\title{
Correlation of the Wells and Simplified Revised Geneva Scores with CT Pulmonary Artery Obstruction Index in Pulmonary Embolism
}

\author{
Morteza Sanei Taheri, ${ }^{1,}{ }^{*}$ Hajar Derakhshandi, ${ }^{2}$ and Marjan Helmi-Khomeirani ${ }^{1}$ \\ ${ }^{1}$ Department of Radiology, Shohada Hospital, Shahid Beheshti University of Medical Sciences, Tehran, Iran \\ ${ }^{2}$ Department of Radiology, Zahedan University of Medical Sciences, Zahedan, Iran \\ Corresponding author: Morteza Sanei Taheri, Department of Radiology, Shohada Hospital, Tajrish Sq, Tehran, Iran. Tel: +98-2122739200, E-mail: saneim@gmail.com
}

Received 2017 January 15; Revised 2017 April 10; Accepted 2017 June 20.

\begin{abstract}
Background: The pulmonary artery obstruction index (PAOI), which is evaluated by the CT pulmonary angiography, offers an objective method to measure the severity of pulmonary arteries obstruction.

Objectives: We decided to investigate the correlation between the PAOI determined by CT pulmonary angiography, as an index to determine the severity of pulmonary embolism (PE), with two clinical models used for predicting the probability of acute PE, namely the Wells criteria and the simplified revised Geneva score.

Patients and Methods: Sixty-four consecutive patients with definite diagnosis of acute PE based on CT pulmonary angiography were enrolled. The Wells score as well as simplified revised Geneva score were calculated retrospectively using the medical records. Then, the PAOI was determined by CT pulmonary angiography. By applying the Pearson's correlation coefficient, the relationships were investigated.

Results: The Wells score had a weak correlation with the PAOI $(r=0.29 ; \mathrm{P}=0.01)$. The simplified revised Geneva score did not have significant correlation with the PAOI $(\mathrm{r}=0.1 ; \mathrm{P}=0.2)$. Furthermore, patients with active cancer had significantly higher $\mathrm{CT}$ indexes than the other patients with $\mathrm{PE}(\mathrm{P}=0.046)$.

Conclusion: Although the Wells and simplified revised Geneva scores are used widely for assessing clinical probability of PE, these scores may not necessarily correlate with the severity of PE. The Wells score had a weak correlation and may somehow predict the severity of PE. Malignancy is a significant risk factor found to be correlated with more severe PE.
\end{abstract}

Keywords: Pulmonary Embolism, Wells Score, Simplified Revised Geneva Score, CT Index, Pulmonary Artery Obstruction Index (PAOI)

\section{Background}

Pulmonary embolism (PE) is a life-threatening disease. Because the clinical signs and symptoms are non-specific or mimic other pathologies, the early diagnosis of acute PE may be difficult (1).

Usually, the clinical diagnosis of PE in suspected patients is not made accurately. According to autopsy results, the prevalence of PE in hospitalized individuals is 15\% - $26 \%$. Although one-third of PEs have contributed to the death of patients, more than $70 \%$ of this number has not been clinically suspected before $(2,3)$. Moreover, about $10 \%$ of patients with PE do not survive after the first embolic occasion. If PE remains neglected, it leads to death in up to $30 \%$ of patients, but this high level can be lowered down to $2 \%$ $10 \%$ if PE is diagnosed and managed timely with anticoagulants (4). This therapy, nevertheless, is not without risk and is accompanied with a complication rate of $10-30 \%$. Therefore, PE needs specific and sensitive diagnostic methods.

Up to the 1990 s, chest X-ray, ventilation/perfusion (V/Q) lung scan, and pulmonary angiography were applied classically to diagnose PE. In general, it should be mentioned that every modality has different advantages and disadvantages with different sensitivity and specificity rates
(5). In the previous decade, several improvements have been made in the diagnosis of PE with minimally invasive modalities such as helical CT and MR imaging. Numerous studies have discussed about the effectiveness of helical CT angiography (CTA) in the diagnosis of acute PE $(1-3,6,7)$.

At the present time, pulmonary CTA is used in suspected patients of PE as the first-line diagnostic approach (8). The pulmonary artery obstruction index (PAOI), which is evaluated by CTA, offers an objective and also reproducible method to measure the severity of pulmonary artery obstruction $(8,9)$. It has remained controversial whether the CT obstruction index indicates mortality in patients with acute PE or not (10).

Assortment of patients suspected to have PE into pretest probability groups helps to select individuals in whom more diagnostic tests are necessary. The original Wells score, which incorporates patients into low, moderate, and high probability groups, is the most widely accepted clinical predictive model. This model is not completely without fault and has been criticized due to the existence of a subjective criterion (i.e., the physician's judgment regarding presence or absence of an alternative diagnosis instead of PE). However, in order to eliminate this restriction, the Geneva group created and verified a com- 
pletely standardized objective rule called the Geneva score revised later. It has been recently simplified into the simplified revised Geneva score $(11,12)$.

\section{Objectives}

The aim of this study was to evaluate the correlation between the Wells and simplified revised Geneva scores with the PAOI calculated by CT pulmonary angiography in PE patients. In other words, we intended to determine whether clinical decision rules for PE are related to the extent of pulmonary emboli (i.e., severity of emboli) seen on CT pulmonary angiography or not.

\section{Patients and Methods}

\subsection{Study Population}

This cross-sectional study was done in our tertiary referral university hospital between January 2014 and June 2015. During this period, 300 patients were clinically suspected to have PE. For all patients, CT pulmonary angiography was performed. Of these, 64 patients with definite diagnosis of PE defined as observing clot on CT pulmonary angiography were included consecutively.

\subsection{Clinical Variables}

A checklist was designed to gather the clinical data required and was filled out by a single physician. Clinical variables included age, gender, clinical symptoms, heart rate, malignancy, deep venous thrombosis (DVT), immobilization history, and recent surgery or fracture. Then, the Wells and simplified revised Geneva scores were measured for each patient. Patients were managed according to a sequential diagnostic strategy including pretest probability of PE assessed by using the original Wells and simplified revised Geneva scores (13). In Wells criteria, seven factors are pointed as presented in Table 1 . PE is likely if the calculated score for the patient is $>4$ and unlikely if $\leq 4$. Table 2 presents factors addressed in the simplified revised Geneva score. Scores of 0 to 2 are considered "unlikely" and scores $\geq 2$ are considered as "likely".

\subsection{CT Pulmonary Angiography Protocol}

All the patients underwent pulmonary contrastenhanced CT scans using a multi-detector 64-row scanner (Philips, Netherland). The images were acquired in the caudocranial direction from the costophrenic angle to 3 $\mathrm{cm}$ above the aortic arch in one breath hold. A total of 130 $\mathrm{mL}$ of low-osmolar contrast agent was injected at a rate of $4 \mathrm{~mL} / \mathrm{sec}$. A scan delay of 16 seconds was used. The images were evaluated by a board-certified radiologist using standard mediastinal windows with real-time ability to change the window and level setting for optimal vessel visualization. The PAOI was determined for each patient, by a single radiologist, from the amount and location of the thrombus on CT images according to the method reported previously $(8,14)$. The pulmonary arteries in the lungs were divided into 10 segmental branches. Three branches were in the upper lobe, two branches in the middle lobe, two branches in the lingula, and five branches in the lower lobe. When thrombi were detected in the proximal pulmonary arteries (i.e., common or lobar arteries), each segment distal to the thrombus was calculated as one point. The total number of segmental arteries yielded the total score. The observation of an isolated thrombus within the segmental artery without any thrombi within the proximal artery was rated as one point. The total score range is between 0 and 40 . Zero means no evidence of thrombus and 40 means thrombosis in both pulmonary arteries.

\subsection{Statistical Analyses}

The data were analyzed by the SPSS software (SPSS Inc. Released 2007. SPSS for Windows, Version 16.0. Chicago, Il). In order to compare the quantitative variables including CT PAOI score, Wells score, and simplified revised Geneva score between the two groups, the independent t test was used. Pearson correlation coefficient was used to report correlation between PAOI score and the clinical decision rules scores. A P value of less than 0.05 was considered statistically significant.

\subsection{Ethics}

The study protocol was approved by the university ethics committee.

\section{Results}

\subsection{Demographics and Clinical Features}

Mean age of the sample was 43.7 years (range, 25 to 73 years). There were 32 males (50\%). Sixteen patients (25\%) had active malignancies. The clinical features of the study population are presented in Table 3. Mean $( \pm$ SD) Wells and simplified revised Geneva scores were 5.14 $( \pm 2.14)$ and 2.97 ( \pm 1.41$)$, respectively.

\subsection{CT Angiography Results}

The mean $( \pm S D)$ CT PAOI was 8.28 ( \pm 5.74$)$. The CT PAOI, Wells score, and simplified revised Geneva score did not show significant difference between males and females ( $\mathrm{P}$ $=0.2$ ). There was a statistically significant correlation between CT PAOI and the presence of malignancy $(\mathrm{P}=0.046)$. 
Table 1. Original Wells Criteria

\begin{tabular}{ll}
\hline Varibales & Point \\
\hline Clinical symptoms of DVT (leg swelling, pain with palpation) & 3.0 \\
\hline Other diagnosis less likely than pulmonary embolism & 3.0 \\
\hline Heart rate $>$ 100 & 1.5 \\
\hline Immobilization $(\geq \mathbf{3}$ days) or surgery in the previous four weeks & 1.5 \\
\hline Previous DVT/PE & 1.5 \\
\hline Hemoptysis & 1.0 \\
\hline Malignancy & 1.0 \\
\hline
\end{tabular}

Abbreviations: DVT, deep vein thrombosis; PE, pulmonary emboli.

Table 2. The Simplified Revised Geneva Score

\begin{tabular}{|l|l|}
\hline Variable & Score \\
\hline Age 65 years or over & 1 \\
\hline Previous DVT or PE & 3 \\
\hline Surgery or fracture within 1 month & 2 \\
\hline Active malignant condition & 3 \\
\hline Unilateral lower limb pain & 2 \\
\hline Hemoptysis & 3 \\
\hline Heart rate 75 to 94 beats per minute & 5 \\
\hline Heart rate 95 or more beats per minute & 4 \\
\hline Pain on deep palpation of lower limb and unilateral edema \\
\hline
\end{tabular}

Abbreviations: DVT, deep vein thrombosis; PE, pulmonary emboli.

Patients with malignancy had higher PAOI scores than patients without cancer. However, CT PAOI did not differ significantly between two groups of patients based on the presence of recent history of immobility/surgery $(\mathrm{P}=0.3)$ or DVT symptoms $(\mathrm{P}=\mathbf{0 . 2 5})$.

The PAOI had statistically significant linear correlation with the Wells score $(r=0.29 ; \mathrm{P}=0.01)$ but was not related to the simplified revised Geneva score $(\mathrm{r}=0.1 ; \mathrm{P}=0.2)$; Figures 1 and 2.

\section{Discussion}

Pulmonary angiography is still the reference standard for PE diagnosis; however, it is invasive, expensive, and occasionally challenging to assess (15). Non-invasive diagnostic modalities have been accepted and different combination of clinical assessment, lower extremity color ultrasonography, D-dimer measurement, V/Q lung scintigraphy and, recently, CT have been considered to eliminate the need for pulmonary angiography. These modalities are used in suspected PE patients in emergency setting or during hospitalization $(16,17)$.

Diagnostic evaluation of suspected PE patients has improved by development of standardized clinical decision rules (CDRs), which show the clinical chance of PE. A combination of normal D-dimer with CDR result of "PE unlikely", can rule out the probability of PE in a great percentage of suspected PE patients (20\% to $40 \%$ ), without requiring additional imaging with CT pulmonary angiography or V/Q scintigraphy. These two radiologic studies include radiation exposure and intravenous contrast or radioisotopes use. Anticoagulants safely are not administered in such patients (17-19). Numerous CDRs, including the data from clinical background and also, physical examination, have been established and confirmed. The Wells criteria consist of six objective variables and one subjective variable which requires physician assessment regarding the chance of a diagnosis, other than PE, for the patient's condition (Table 1) (17). Conversely, the more recently revised Geneva score incorporates eight objective clinical variables (12).

In a recent meta-analysis, the reliability of the scores 
Table 3. Clinical Characteristics Used for Clinical Decision Rules in Pulmonary Embolism Among 64 Patients with Embolus Observed on CT Pulmonary Angiography

\begin{tabular}{|c|c|}
\hline Characteristics & No. (\%) \\
\hline Age $\geq 65$ years & $20(31.3)$ \\
\hline Previous DVT/pulmonary embolism history & $4(6.3)$ \\
\hline Surgery/fracture history within 1 month & $36(56.3)$ \\
\hline Active malignancy & $16(25)$ \\
\hline Unilateral lower limb pain & $16(25)$ \\
\hline Hemoptysis & 0 \\
\hline \multicolumn{2}{|l|}{ Heart rate, beats per min } \\
\hline$\geq 95$ & $12(18.8)$ \\
\hline 75 to 94 & $42(65.6)$ \\
\hline$\leq 74$ & $10(15.6)$ \\
\hline Pain on lower limb deep palpation and unilateral edema & $14(21.9)$ \\
\hline Clinical symptoms of DVT & $22(34.4)$ \\
\hline Recent immobilization/surgery & $38(59.4)$ \\
\hline Other diagnosis less likely than PE & $52(81.3)$ \\
\hline
\end{tabular}

Abbreviations: DVT, deep vein thrombosis; PE, pulmonary emboli.
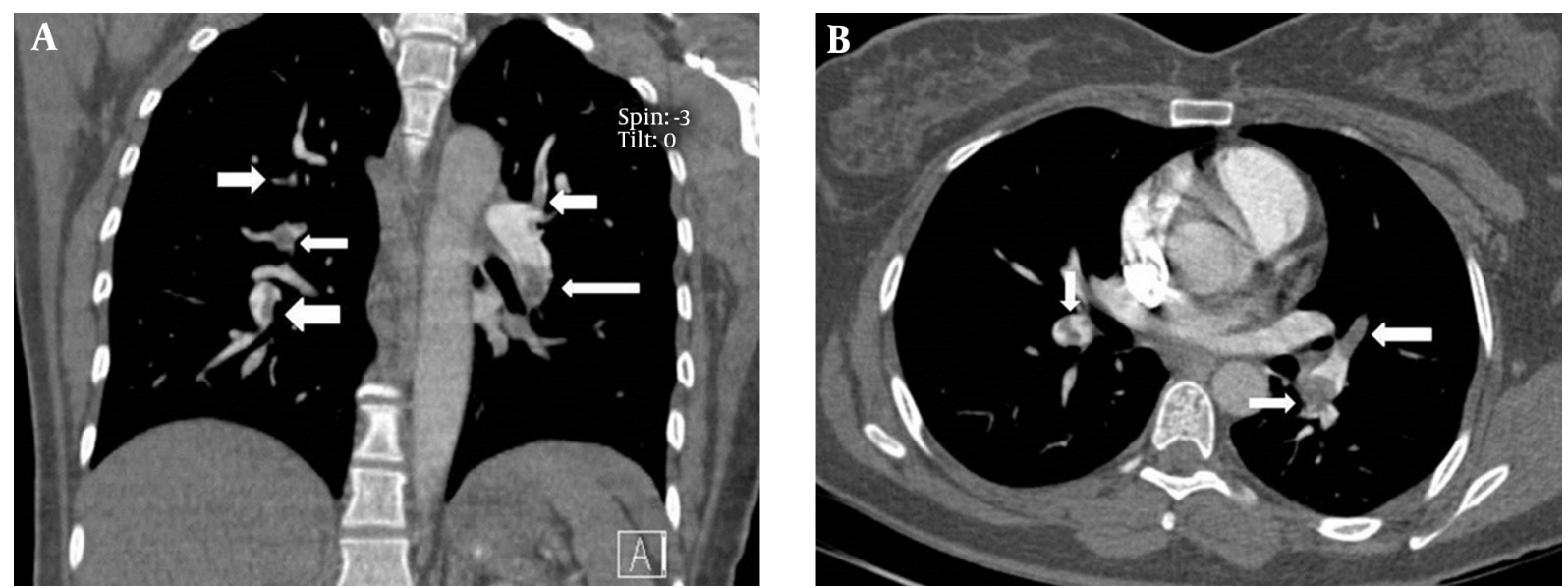

Figure 1. A, Coronal and B, Axial CT angiography images in a 40-year-old female show filling defects (arrows). The pulmonary artery obstruction index (PAOI) was 23 .

of Wells score and the revised Geneva score was examined and compared by evaluating the prevalence of the PE in any group of the clinical probability and was demonstrated to be similar (20). In the present study, the simplified Geneva score had a strong correlation with the Well's score results. This in agreement with a former study (20).

Douma et al. (13) evaluated the accuracy of four clinical probability scores (revised Geneva score, Wells rule, simplified revised Geneva score, and simplified Wells rule) in combination with D-dimer testing, in order to rule out acute PE in 807 suspected patients. They claimed that four scores indicated similar efficiency for the exclusion of acute PE when associated with a normal D-dimer level. Comparing of the predictive precision and concordance of the Wells and Geneva rules showed similar predictive accuracies for PE. It should be mentioned that the Wells criteria is more rapid, simple, and economical, and can also offer outcomes, comparable to those of the Geneva criteria.

The results of the current investigation are consistent with those of the study conducted by Attia et al., which reported that the PAOI is correlated with dyspnea and tachycardia; however, they did not show any relationship with 

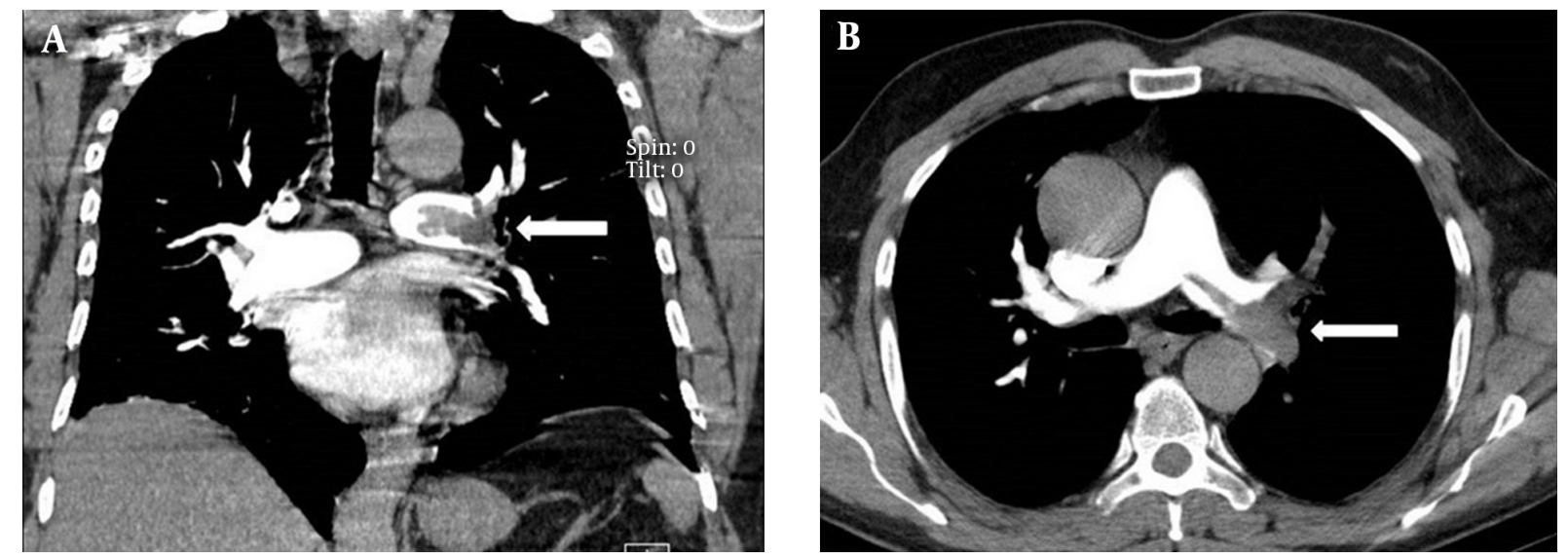

Figure 2. A, Coronal and B, Axial CT angiography images in a 50-year-old female show filling defects (arrows). The pulmonary artery obstruction index (PAOI) was 11.

hemoptysis and chest pain, which are common in peripheral pulmonary embolisms (21). According to our results, Wells score can be used to evaluate the severity of PE similar to CT index.

Right ventricular (RV) malfunction and circulatory collapse are the main causes of death in patients with severe PE that often happen within the first hours after admission (22). Patients with RV failure have a poorer prognosis compared to individuals with normal RV function. Accordingly, identifying RV malfunctions following PE is beneficial for risk stratification and also, the better selection of therapeutic approach (23).

The CT obstruction index is an objective and reproducible method that measures the severity and extent of thrombosis within the pulmonary arteries from PE(8). Several reports have exhibited that the PAOI correlates with the RV malfunction. Some studies have demonstrated that CT can not only certainly and perfectly diagnose acute PE, but can also quantify the severity of $\operatorname{PE}(24,25)$.

In this study, patients with malignancy had significantly higher CT indexes that confirm more severe forms of PE in such patients. Therefore, when the signs and symptoms of PE appear in patients with active cancer, we should expect a more severe and even a lethal PE compared to other high-risk patients and should be aware about the imminent RV failure or circulatory collapse. Such association was not found for other predisposing factors including recent surgery or immobilization.

Due to incomplete data and resources, we were not able to follow the patients to find correlation between the burden of PE and prognosis of the patients.

Given that PE does not have any distinctive and specific diagnostic signs, and since the earlier diagnosis of PE decreases mortality rate, the pulmonary CTA can be a helpful diagnostic method with high sensitivity for diagnosis. In the current study, we indicated the correlation of CT index and Wells Scores, and this association can be applied in early evaluation of the PE severity with Wells score. On the other hand, patients with active malignancy had higher CT indexes that confirm the more severity of PE in these patients and emphasis on the more consideration of lethal complications in such patients. These findings help physicians to better and timely selection of early treatment approach so that reduce costs and mortality rate. However, further more evaluations are needed to establish communication between Wells and simplified revised Geneva Scores and CT obstruction index.

\section{Footnotes}

Authors' Contributions: Morteza Sanei Taheri proposed the concept, designed and supervised the study, and revised the final article. Hajar Derakhshandi collected data and reviewed the literature. Marjan Helmi-Khomeirani drafted the article and reviewed the literature.

Financial Disclosure: No financial disclosure to declare.

Funding/Support: This study was supported by School of Medicine, Shahid Beheshti University of Medical Sciences, Tehran, Iran.

\section{References}

1. Fedullo PF, Tapson VF. Clinical practice. The evaluation of suspected pulmonary embolism. NEngl J Med. 2003;349(13):1247-56. doi: 10.1056/NEJMcp035442. [PubMed:14507950].

2. Moser KM. Venous thromboembolism. Am Rev Respir Dis. 1990;141(1):235-49. doi: 10.1164/ajrccm/141.1.235. [PubMed: 2404439]. 
3. Stein PD, Henry JW. Prevalence of acute pulmonary embolism among patients in a general hospital and at autopsy. Chest. 1995;108(4):97881. doi: 10.1378/chest.108.4.978. [PubMed: 7555172].

4. Roy PM, Meyer G, Vielle B, Le Gall C, Verschuren F, Carpentier F, et al. Appropriateness of diagnostic management and outcomes of suspected pulmonary embolism. Ann Intern Med. 2006;144(3):157-64. doi: 10.7326/0003-4819-144-3-200602070-00003. [PubMed: 16461959].

5. Ghaye B, Remy J, Remy-Jardin M. Non-traumatic thoracic emergencies: CT diagnosis of acute pulmonary embolism: the first 10 years. Eur Radiol. 2002;12(8):1886-905. doi: 10.1007/s00330-002-1506z. [PubMed: 12136308].

6. Miron MJ, Perrier A, Bounameaux H, de Moerloose P, Slosman DO, Didier D, et al. Contribution of noninvasive evaluation to the diagnosis of pulmonary embolism in hospitalized patients. Eur Respir J. 1999;13(6):1365-70. doi: 10.1183/09031936.99.13613719. [PubMed: 10445613].

7. Goldhaber SZ, Elliott CG. Acute pulmonary embolism: part I: epidemiology, pathophysiology, and diagnosis. Circulation. 2003;108(22):2726-9. doi: 10.1161/01.CIR.0000097829.89204.0C [PubMed: 14656907].

8. Qanadli SD, El Hajjam M, Vieillard-Baron A, Joseph T, Mesurolle B, Oliva VL, et al. New CT index to quantify arterial obstruction in pulmonary embolism: comparison with angiographic index and echocardiography. AJR Am J Roentgenol. 2001;176(6):1415-20. doi: 10.2214/ajr.176.6.1761415. [PubMed: 11373204]

9. Erdur B, Karabulut N, Turkcuer I, Ergin A. Diagnostic work-up of patients with suspected pulmonary embolism: a survey of strategies used by emergency physicians. Diagn Interv Radiol. 2009;15(3):166-71. [PubMed: 19728260].

10. Araoz PA, Gotway MB, Trowbridge RL, Bailey RA, Auerbach AD, Reddy GP, et al. Helical CT pulmonary angiography predictors of in-hospital morbidity and mortality in patients with acute pulmonary embolism. J Thorac Imaging. 2003;18(4):207-16. doi: 10.1097/00005382200310000-00001. [PubMed: 14561905].

11. Klok FA, Mos IC, Nijkeuter M, Righini M, Perrier A, Le Gal G, et al. Simplification of the revised Geneva score for assessing clinical probability of pulmonary embolism. Arch Intern Med. 2008;168(19):2131-6. doi: 10.1001/archinte.168.19.2131. [PubMed: 18955643].

12. Le Gal G, Righini M, Roy PM, Sanchez O, Aujesky D, Bounameaux H, et al. Prediction of pulmonary embolism in the emergency department: the revised Geneva score. Ann Intern Med. 2006;144(3):165-71. doi: 10.7326/0003-4819-144-3-200602070-00004. [PubMed:16461960].

13. Douma RA, Mos IC, Erkens PM, Nizet TA, Durian MF, Hovens MM, et al. Performance of 4 clinical decision rules in the diagnostic management of acute pulmonary embolism: a prospective cohort study. Ann Intern Med. 2011;154(11):709-18. doi: 10.7326/0003-4819-15411-201106070-00002. [PubMed: 21646554].

14. Inonu H, Acu B, Pazarli AC, Doruk S, Erkorkmaz U, Altunkas A. The value of the computed tomographic obstruction index in the identification of massive pulmonary thromboembolism. Diagn Interv Radiol. 2012;18(3):255-60. doi: 10.4261/1305-3825.DIR.4597-11.4. [PubMed: 22249888].

15. van Beek EJ, Brouwerst EM, Song B, Stein PD, Oudkerk M. Clinical va- lidity of a normal pulmonary angiogram in patients with suspected pulmonary embolism-a critical review. Clin Radiol. 2001;56(10):83842. doi: 10.1053/crad.2001.0778. [PubMed: 11895301].

16. Kline JA, Webb WB, Jones AE, Hernandez-Nino J. Impact of a rapid rule-out protocol for pulmonary embolism on the rate of screening, missed cases, and pulmonary vascular imaging in an urban US emergency department. Ann Emerg Med. 2004;44(5):490-502. doi: 10.1016/S0196064404002872. [PubMed: 15520709].

17. Wells PS, Anderson DR, Rodger M, Stiell I, Dreyer JF, Barnes D, et al. Excluding pulmonary embolism at the bedside without diagnostic imaging: management of patients with suspected pulmonary embolism presenting to the emergency department by using a simple clinical model and d-dimer. Ann Intern Med. 2001;135(2):98-107. doi 10.7326/0003-4819-135-2-200107170-00010. [PubMed: 11453709].

18. van Belle A, Buller HR, Huisman MV, Huisman PM, Kaasjager K, Kamphuisen PW, et al. Effectiveness of managing suspected pulmonary embolism using an algorithm combining clinical probability, D dimer testing, and computed tomography. JAMA. 2006;295(2):172-9. doi: 10.1001/jama.295.2.172. [PubMed: 16403929].

19. Wells PS, Anderson DR, Rodger M, Ginsberg JS, Kearon C, Gent M, et al Derivation of a simple clinical model to categorize patients probability of pulmonary embolism: increasing the models utility with the SimpliRED D-dimer. Thromb Haemost. 2000;83(3):416-20. [PubMed: 10744147].

20. Roy PM, Colombet I, Durieux P, Chatellier G, Sors H, Meyer G. Systematic review and meta-analysis of strategies for the diagnosis of suspected pulmonary embolism. BMJ. 2005;331(7511):259. doi: 10.1136/bmj.331.7511.259. [PubMed: 16052017].

21. Attia NM, Seifeldein GS, Hasan AA, Hasan A. Evaluation of acute pulmonary embolism by sixty-four slice multidetector CT angiography: Correlation between obstruction index, right ventricular dysfunction and clinical presentation. Egypt J Radiol Nucl Med. 2015;46(1):2532. doi: 10.1016/j.ejrnm.2014.10.007.

22. Wood KE. Major pulmonary embolism: review of a pathophysiologic approach to the golden hour of hemodynamically significant pulmonary embolism. Chest. 2002;121(3):877-905. [PubMed: 11888976].

23. Torbicki A, Perrier A, Konstantinides S, Agnelli G, Galie N, Pruszczyk $\mathrm{P}$, et al. Guidelines on the diagnosis and management of acute pulmonary embolism: the Task Force for the Diagnosis and Management of Acute Pulmonary Embolism of the European Society of Cardiology (ESC). Eur Heart J. 2008;29(18):2276-315. doi: 10.1093/eurheartj/ehn310. [PubMed: 18757870].

24. van der Meer RW, Pattynama PM, van Strijen MJ, van den BergHuijsmans AA, Hartmann IJ, Putter H, et al. Right ventricular dysfunction and pulmonary obstruction index at helical CT: prediction of clinical outcome during 3-month follow-up in patients with acute pulmonary embolism. Radiology. 2005;235(3):798-803. doi: 10.1148/radiol.2353040593. [PubMed: 15845793].

25. Zhao DJ, Ma DQ, He W, Wang JJ, Xu Y, Guan CS. Cardiovascular parameters to assess the severity of acute pulmonary embolism with computed tomography. Acta Radiol. 2010;51(4):413-9. doi: 10.3109/02841851003649266. [PubMed: 20380604]. 